\title{
Effects of Caryota mitis profilin-loaded PLGA nanoparticles in a murine model of allergic asthma
}

This article was published in the following Dove Press journal:

International Journal of Nanomedicine

22 November 2013

Number of times this article has been viewed

\section{Xiaojun Xiao ${ }^{1, *}$ \\ Xiaowei Zeng ${ }^{2, *}$ \\ Xinxin Zhang ${ }^{3, *}$ \\ $\mathrm{Li} \mathrm{Ma}{ }^{3}$ \\ Xiaoyu Liu' \\ Haiqiong $\mathrm{Yu}^{\prime}$ \\ Lin $\mathrm{Mei}^{2}$ \\ Zhigang Liu'}

'Institute of Allergy and Immunology, School of Medicine, Shenzhen

University, Shenzhen, ${ }^{2}$ Division of Life and Health Sciences, Graduate School at Shenzhen, Tsinghua University, Shenzhen, ${ }^{3}$ Faculty of Basic Medical Science, Nanchang University, Nanchang, People's Republic of China

*These authors contributed equally to this work
Correspondence: Zhigang Liu

School of Medicine, Shenzhen University, Shenzhen 518060, Guangdong,

People's Republic of China

Fax +86755 26534902

Email lzg@szu.edu.cn

Lin Mei

L40I, Tsinghua Campus, Xili University

Town, Shenzhen 5I8055, Guangdong,

People's Republic of China

Fax +8675526036736

Email mei.lin@sz.tsinghua.edu.cn
Background: Pollen allergy is the most common allergic disease. However, tropical pollens, such as those of Palmae, have seldom been investigated compared with the specific immunotherapy studies done on hyperallergenic birch, olive, and ragweed pollens. Although poly(lacticco-glycolic acid) (PLGA) has been extensively applied as a biodegradable polymer in medical devices, it has rarely been utilized as a vaccine adjuvant to prevent and treat allergic disease. In this study, we investigated the immunotherapeutic effects of recombinant Caryota mitis profilin (rCmP)-loaded PLGA nanoparticles and the underlying mechanisms involved.

Methods: A mouse model of allergenic asthma was established for specific immunotherapy using rCmP-loaded PLGA nanoparticles as the adjuvant. The model was evaluated by determining airway hyperresponsiveness and levels of serum-specific antibodies (IgE, IgG, and IgG2a) and cytokines, and observing histologic sections of lung tissue.

Results: The rCmP-loaded PLGA nanoparticles effectively inhibited generation of specific $\operatorname{IgE}$ and secretion of the Th2 cytokine interleukin-4, facilitated generation of specific $\operatorname{IgG} 2 \mathrm{a}$ and secretion of the Th1 cytokine interferon-gamma, converted the Th2 response to Th1, and evidently alleviated allergic symptoms.

Conclusion: PLGA functions more appropriately as a specific immunotherapy adjuvant for allergen vaccines than does conventional $\mathrm{Al}(\mathrm{OH})_{3}$ due to its superior efficacy, longer potency, and markedly fewer side effects. The rCmP-loaded PLGA nanoparticles developed herein offer a promising avenue for specific immunotherapy in allergic asthma.

Keywords: nanoparticles, Caryota mitis profilin, PLGA, allergic asthma, adjuvant

\section{Introduction}

Allergic disease is frequently encountered, is classified as one of the four common noncommunicable diseases by the World Health Organization, ${ }^{1}$ and significantly jeopardizes human health. The incidence of allergic disease is $30 \%-40 \%$ worldwide, has doubled in the last 30 years, and is still increasing at a rate higher than $1 \%$ annually. ${ }^{2,3}$ Patients effectively treated by allopathy, including hormones and antihistamines, are at risk of side effects, aggravated metabolic burden, and disease recurrence in the event of termination of medication, that leads to a vicious cycle. ${ }^{4,5}$ As a sole treatment for allergic disease, specific immunotherapy functions to regulate the Th1/Th2 equilibrium, but has short-term efficacy, ${ }^{6,7}$ poor stability, and discernible side effects that limit its clinical use. ${ }^{8}$ Therefore, it is important to identify other secure and more stable treatment methods, the mechanisms of which are of crucial theoretical and practical significance.

Vaccination is among the most important medical interventions that have helped to reduce and eliminate a number of diseases. The development of nanoparticles has 
started to receive a lot of attention in order to provide effective immunization through better targeting and by triggering an antibody response at the cellular level. Nanotechnology has also helped to formulate efficient vaccine delivery systems that can prevent the encapsulated antigen from damage in the in vivo environment and can maintain sustained release that helps to induce the immunostimulatory properties of the vaccine. ${ }^{9,10}$ With very few adjuvants currently being used in marketed human vaccines, a critical need exists for novel immunopotentiators and delivery vehicles capable of eliciting humoral and cellular immunity. ${ }^{9}$ Poly(lactic-co-glycolic acid) (PLGA), a biocompatible degradable material approved by the US Food and Drug Administration, has been used extensively in bioabsorbable sutures, orthopedic fixation and tissue repair materials, and controlled drug delivery systems. ${ }^{11,12}$ PLGA and its derivatives have been spotlighted as carriers in the controlled drug delivery field. ${ }^{13}$ For instance, inorganic biodegradable polymer nanohybrid materials have been prepared by combining inorganic nanoparticles with PLGA materials, and have been used to develop microcapsules that release drugs slowly. ${ }^{14,15}$ PLGA prevents nucleic acids, peptide chains, and proteins from degradation by encapsulation, allowing it to be applied as an adjuvant in antiallergic, hepatitis B, and human immunodeficiency virus vaccines. Scholl et al verified that the allergen slowly released from PLGA microcapsules effectively induced generation of antibody IgG. ${ }^{16}$ Profilins are ubiquitous proteins, present in all eukaryotic cells and identified as allergens in pollen, latex, and plant foods. Purified natural and recombinant profilins for in vitro and in vivo allergy tests are helpful in the diagnostic work-up. Recombinant birch profilin, as well as natural profilins from birch, timothy grass, and mugwort elicit IgEmediated histamine release from basophils of pollen-allergic patients which cause type I allergy symptoms. However, there has been no report on the use of recombinant Caryota mitis profilin (rCmP) in the treatment of asthma. ${ }^{17}$ Thus, in this study, we encapsulated rCmP into PLGA nanoparticles. The immune protective, preventive, and treatment effects of rCmP-loaded PLGA nanoparticles in mouse asthma as well as the relevant mechanisms were investigated, which will be innovative in immunotherapy for allergic asthma.

\section{Materials and methods}

Animals and materials

Female BALB/c mice (specific pathogen-free grade, aged 5-6 weeks, weight 16-20 g) were purchased from the Animal Center of Guangdong Province and fed in a specific pathogenfree grade breeding room. rCmP, PLGA (lactide:glycolide
50:50, molecular weight 24,000-38,000) and polyvinyl alcohol (80\% hydrolyzed) were obtained from Sigma-Aldrich (St Louis, MO, USA). Analytical grade dichloromethane obtained from J\&K Chemical Co, Ltd (Beijing, People's Republic of China) was used without any further purification. $\mathrm{Al}(\mathrm{OH})_{3}$ and methacholine were also purchased from SigmaAldrich. Biotin-labeled goat anti-mouse IgE and horseradish peroxidase (HPR)-labeled goat anti-mouse IgG and IgG2a were from eBioscience (San Diego, CA, USA). Mouse interleukin (IL)-4, IL-10, and interferon gamma (IFN- $\gamma$ ) enzyme-linked immunosorbent assay (ELISA) kits were obtained from BioLegend (San Diego, CA, USA).

\section{Expression and purification of $\mathrm{rCmP}$}

The strain was expressed by the gene sequences BL21 of $\mathrm{rCmP}$ at $37^{\circ} \mathrm{C}$ in a shaking water bath with a shaking speed of $120 \mathrm{rpm}$, which has been described previously. ${ }^{18}$ The protein was purified with a $\mathrm{Ni}^{2+}$ affinity chromatography column. The elution, which including resultant protein was dialyzed and lyophilized.

\section{Formation of rCmP-loaded nanoparticles}

rCmP-loaded PLGA nanoparticles were prepared by a w/o/w double emulsion solvent evaporation method as reported by Jain et al with slight modifications. ${ }^{19}$ Briefly, aqueous phase $(0.5 \mathrm{~mL})$ containing $10 \mathrm{mg}$ of $\mathrm{rCmP}$ was added to organic phase $(2 \mathrm{~mL} /$ dichloromethane and acetone at a volume ratio 9:1) including copolymer PLGA $(5 \% \mathrm{w} / \mathrm{v})$. The primary emulsion (w/o) was obtained by probe sonication in an ice bath for 120 seconds. The primary emulsion was then added to $100 \mathrm{~mL}$ of aqueous solution containing polyvinyl alcohol $(1 \% \mathrm{w} / \mathrm{v})$ as an emulsifier and sonicated in an ice bath for 60 seconds to form the secondary emulsion $(\mathrm{w} / \mathrm{o} / \mathrm{w})$. The resulting emulsion was stirred vigorously overnight to evaporate the organic phase. The nanoparticles were collected by centrifugation $\left(20,000 \mathrm{rpm}, 15\right.$ minutes, $\left.4^{\circ} \mathrm{C}\right)$, washed twice with distilled water to remove residual polyvinyl alcohol, and subjected to lyophilization. The final nanoparticles were stored at $4^{\circ} \mathrm{C}$ in a refrigerator.

\section{Characterization of $\mathrm{rCmP}$-loaded nanoparticles \\ Size and zeta potential}

The nanoparticle size and size distribution were measured by dynamic light scattering using a Mastersizer 2000 (Malvern Instruments Ltd, Malvern, UK). Before measurement, approximately $0.2 \mathrm{mg}$ of freshly prepared nanoparticles were diluted in $1 \mathrm{~mL}$ of distilled water and sonicated in an ice bath 
for 30 seconds. The data obtained represent the average of three measurements.

\section{Surface morphology}

The surface morphology of the nanoparticles was examined by field emission scanning electron microscopy (FESEM) using a JSM-6700F system (JEOL, Tokyo, Japan) operated at a $5.0 \mathrm{kV}$ accelerating voltage. To prepare samples for FESEM, the particles were fixed on the stub by double-sided sticky tape and then coated with a platinum layer using an automatic fine platinum coater (JFC-1300, JEOL) for 60 seconds.

\section{Protein loading and encapsulation efficiency}

The protein loading content (LC) and encapsulation efficiency (EE) of the nanoparticles were determined by bicinchoninic acid protein assay. In summary, $5 \mathrm{mg}$ of nanoparticles were dissolved in $2 \mathrm{~mL}$ of aqueous phase containing $1 \%$ sodium dodecyl sulfate and $0.05 \mathrm{M}$ sodium hydroxide $(\mathrm{NaOH})$. Each batch was performed in triplicate. The protein LC and the EE of the rCmP-loaded nanoparticles were calculated using the following equations: ${ }^{20}$

$$
\begin{aligned}
& \text { LC }(\%)=\frac{\text { Weight of protein in the nanoparticles }}{\text { Weight of the nanoparticles }} \times 100 \% \\
& \mathrm{EE}(\%)=\frac{\text { Weight of protein in the nanoparticles }}{\text { Weight of the feeding protein }} \times 100 \%
\end{aligned}
$$

In vitro release study

To perform the in vitro release study, $20 \mathrm{mg}$ of rCmP-loaded nanoparticles was dispersed in $5 \mathrm{~mL}$ of phosphate-buffered saline (PBS, $\mathrm{pH} 7.4$ ) to form a suspension. The suspension was incubated at $37^{\circ} \mathrm{C}$ in a shaking water bath at a shaking frequency of $120 \mathrm{rpm}$. At appropriate time intervals, a $1 \mathrm{~mL}$ sample was collected following centrifugation at 20,000 rpm for 30 minutes and replaced with fresh PBS. Samples were detected using the bicinchoninic acid assay method to determine the fraction of protein released. Each batch was repeated in triplicate.

\section{SDS-PAGE and Western blotting of $\mathrm{rCmP}$-loaded nanoparticles}

The protein components in $\mathrm{rCmP}$ and in the rCmP-loaded PLGA nanoparticles (PLGA-rCmP) were subjected to sodium dodecyl sulfate polyacrylamide gel electrophoresis (SDS-PAGE) identification and electroblotted onto nitrocellulose membranes. The membranes were blocked with $3 \%$ bovine serum albumin (BSA) overnight at $4^{\circ} \mathrm{C}$ and then incubated in diluted (1:5) positive serum of allergic patients at $37^{\circ} \mathrm{C}$ for 2 hours.

Biotin-labeled goat anti-human IgE diluted $(1: 3,000)$ by TBST (TBS/0.05\% Tween-20) was added to the resulting membranes, which were then incubated at $37^{\circ} \mathrm{C}$ for 2 hours. After adding HRP-labeled streptavidin diluted $(1: 1,000)$ by TBST, the membranes were incubated at $37^{\circ} \mathrm{C}$ for another 1.5 hours. The membranes were washed three times after each step ( 5 minutes). They were then visualized in freshly prepared diaminobenzidine substrate solution and observed after being washed in double-distilled water.

\section{Animal sensitization and specific immunotherapy}

The administrative committee on animal research at the Graduate School of Shenzhen, Tsinghua University, approved all the protocols used for the animal experiments. The animal sensitization model and specific immunotherapy were designed (Figure 1) by combining the previous literature and the requirements herein. ${ }^{21-24}$ The mice were randomly divided into five groups $(\mathrm{n}=8)$, ie, a normal group, a model group, a $\mathrm{PLGA}-\mathrm{rCmP}$ group, a $\mathrm{rCmP} / \mathrm{Al}(\mathrm{OH})_{3}$ (ie, $\mathrm{rCmP}$ ) vaccine group, and a blank PLGA nanoparticle control group. Mice in the normal group were sensitized and treated with normal saline, and the other groups were sensitized three times every

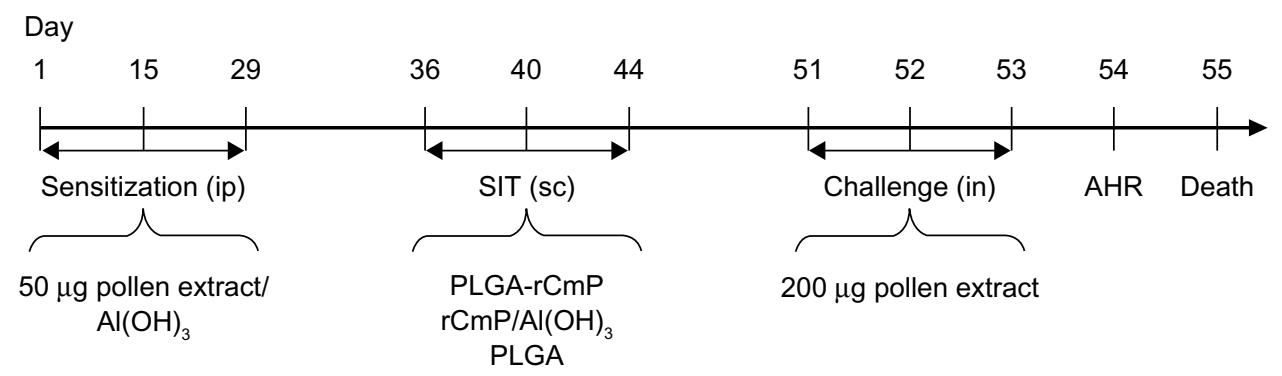

Figure I Experimental design. Five groups of mice ( $\mathrm{n}=\mathbf{8}$ per group) were used in the experiment.

Abbreviations: AHR, airway hyperresponsiveness; PLGA, poly(lactic-co-glycolic acid); rCmP, recombinant Caryota mitis profilin; SIT, specific immunotherapy; ip, intraperitoneal injection; sc, subcutaneous injection; in, nasal inhalation. 
3 weeks by intraperitoneal injection of $50 \mu \mathrm{g}$ Caryota mitis $(\mathrm{Cm})$ pollen crude proteins adsorbed on $2 \mathrm{mg}$ of $\mathrm{Al}(\mathrm{OH})_{3}$. The treatment was initiated on day 36. The PLGA-rCmP group was subcutaneously injected with nanoparticles containing $50 \mu \mathrm{g} \mathrm{rCmP}$, the $\mathrm{rCmP}$ group was injected with $\mathrm{rCmP}$ adsorbed on $2 \mathrm{mg}$ of $\mathrm{Al}(\mathrm{OH})_{3}$, and the PLGA group was injected with an equivalent amount of PLGA. The mice were treated three times every 4 weeks. The mice were intranasally challenged three times daily with $200 \mu \mathrm{g}$ of crude proteins one week after the final treatment. The mice were subjected to an airway hyperresponsiveness (AHR) test 24 hours after the final challenge and were then euthanized.

\section{Assessment of AHR to methacholine challenge}

Twenty-four hours after the final challenge, AHR was measured using unrestrained whole-body plethysmography with a four-chamber system (Buxco Research Systems, Wilmington, NC, USA). The baseline response was recorded for 5 minutes in the event of stabilized respiration after 10 minutes of adaptation. The responses of the mice were then recorded every 5 minutes after inhaling different concentrations of atomized methacholine solutions $(0,6.25,12.5,25,50$, and $100 \mathrm{mg} / \mathrm{mL}$ PBS). Tests at two different concentrations were separated temporarily to allow the respiratory intensity to drop back to baseline. The percentage curves for Penh values at different methacholine concentrations were plotted based on that of PBS.

\section{Determination of allergen-specific IgE, $\lg \mathrm{G}$, and $\lg \mathrm{g} 2 \mathrm{a}$ antibodies in serum}

Blood was sampled from mouse eyes 24 hours after the AHR test, left undisturbed for 2 hours, and stored overnight at $4^{\circ} \mathrm{C}$. The blood was then centrifuged at $4^{\circ} \mathrm{C}$ and $3,000 \mathrm{rpm}$ for 10 minutes, and the supernatant serum was collected. Next, the responses of C. mitis pollen-specific IgE, $\operatorname{IgG}$, and IgG2a were detected in serum using the indirect ELISA protocol. The antibodies were coated with $1 \mathrm{ng} / \mu \mathrm{g} \mathrm{rCmP}$ and stored overnight at $4{ }^{\circ} \mathrm{C}$. The plates, which were washed with PBST and blocked with 3\% BSA/PBS (200 $\mu \mathrm{L} /$ well), were incubated at $37^{\circ} \mathrm{C}$ for 2 hours. Mouse serum diluted ( $\mathrm{IgG}$, 1:2,000; IgG2a, 1:1,000; IgE, 1:5) by 1\% BSA-PBST and $1 \%$ BSA-PBST was added to the test wells and the control well $(100 \mu \mathrm{L} /$ well $)$ after the plates were washed with PBST, and incubated at $37^{\circ} \mathrm{C}$ for one hour. Next, $100 \mu \mathrm{L}$ of biotinlabeled goat anti-mouse secondary antibodies diluted (HRPIgG, 1:2,000; HRP-IgG2a, 1:2,000; biotin-IgE, 1:20,000) with $1 \% \mathrm{BSA} / \mathrm{PBST}$ were added after the plates were washed with PBST, and incubated at $37^{\circ} \mathrm{C}$ for a further hour. The plates were subjected to color development in the dark at $37^{\circ} \mathrm{C}$ for 10 minutes utilizing $3,3^{\prime}, 5,5^{\prime}$-tetramethylbenzidine solution after being washed with PBST, and the reactions were terminated by $1 \mathrm{M} \mathrm{H}_{2} \mathrm{SO}_{4}$. The absorbances at $450 \mathrm{~nm}$ were then measured on a microplate reader.

\section{Bronchoalveolar lavage and inflammatory cell differential counting}

Immediately after being euthanized, the mice were subjected to bronchoalveolar lavage. The lung was immediately lavaged via the trachea cannula with $0.8 \mathrm{ml}$ of PBS. The bronchoalveolar lavage fluid was collected after five bouts of pumping. Each mouse underwent bronchoalveolar lavage three times, yielding approximately $2 \mathrm{~mL}$ of bronchoalveolar lavage fluid each time (recovery rate $>80 \%$ ). The combined bronchoalveolar lavage fluid was centrifuged at $4^{\circ} \mathrm{C}$ for 10 minutes, and the supernatant was then sampled to determine cytokine levels. The precipitate was resuspended with $100 \mu \mathrm{L}$ of PBS, which was utilized for cell counting $(10 \mu \mathrm{L})$ and cell differential counting $(90 \mu \mathrm{L})$. The stained cells were then classified and counted using Liu's stain. At least 200 cells were counted and classified as neutrophils, eosinophils, lymphocytes, or macrophages according to their morphologic characteristics.

\section{Cytokine assay}

Mouse spleen tissue was ground and filtered through a 200-mesh screen. The splenocytes, which were then resuspended by Roswell Park Memorial Institute 1640 medium (100 mg/L fetal bovine serum, $100 \mathrm{U} / \mathrm{L}$ penicillin, and $100 \mathrm{mg} / \mathrm{L}$ streptomycin) and erythrocyte lysate were collected by centrifugation. The cells were cultured in Roswell Park Memorial Institute 1640 medium in 24-well plates at a density of $5 \times 10^{6} /$ well, stimulated with $C$. mitis pollen crude proteins $(200 \mu \mathrm{g} / \mathrm{well})$, and incubated at $37^{\circ} \mathrm{C}$ for 72 hours. The culture medium was then collected and centrifuged at $4^{\circ} \mathrm{C}$ and 1,500 rpm. The supernatant was then collected and stored at $-80^{\circ} \mathrm{C}$ for cytokine determination. The IL-4, IL-10, and INF- $\gamma$ levels in the supernatants of the splenocyte culture medium and bronchoalveolar lavage fluid were measured strictly according to the instructions of the ELISA kit (Biolegend, San Diego, CA, USA).

\section{Lung histology and inflammation scoring}

The mouse lungs were immediately removed and fixed in cold formalin solution for 24 hours, and then dehydrated by different concentrations of ethanol. The lung tissues 
were embedded in paraffin and cut into approximately $5 \mathrm{~mm} \times 5 \mathrm{~mm}$ blocks, which were sliced using a slicing machine ( $4 \mu \mathrm{m}$ thick). The slices were then stained with hematoxylin and eosin and sealed to observe any histologic changes under an optical microscope.

\section{Statistical analysis}

The data are expressed as the mean \pm standard deviation. Statistical comparisons between two groups were made using the two-tailed, unpaired Student's $t$-test with Statistical Package for the Social Sciences version 16 software (SPSS Inc, Chicago, IL, USA). $P<0.05$ was considered to be statistically significant.

\section{Results}

\section{Characterization of nanoparticles}

The size and size distribution of the rCmP-loaded PLGA nanoparticles were investigated by dynamic light scattering. Physicochemical characteristics, such as particle size and surface properties, play an important role in vitro and in vivo. Therefore, they affect the therapeutic efficacy of specific immunotherapy. ${ }^{25}$ The mean diameter of the rCmP-loaded PLGA nanoparticles was approximately $180 \mathrm{~nm}$, which is in the excellent size range for cellular uptake of nanoparticles. ${ }^{26}$ The nanoparticles exhibited a relatively narrow polydispersity index $(\approx 0.135)$, which is useful for treatment effects. The zeta potential of nanoparticles is a crucial factor for stability in a suspension through the electrostatic repulsion between the nanoparticles, interaction with the cell membrane in vivo, and judgment of component onto the nanoparticle surface. ${ }^{27}$ The rCmP-loaded PLGA nanoparticles showed a negative surface charge of around $-10 \mathrm{mV}$ which was stable in vivo. The protein LC and EE of the rCmP-loaded PLGA nanoparticles was $9.79 \%$ and $63.56 \%$, respectively.

The surface morphology of the rCmP-loaded PLGA nanoparticles was investigated by FESEM. Figure 2A shows an FESEM image for rCmP-loaded PLGA nanoparticles. The nanoparticles seemed to be about $180 \mathrm{~nm}$ in diameter and

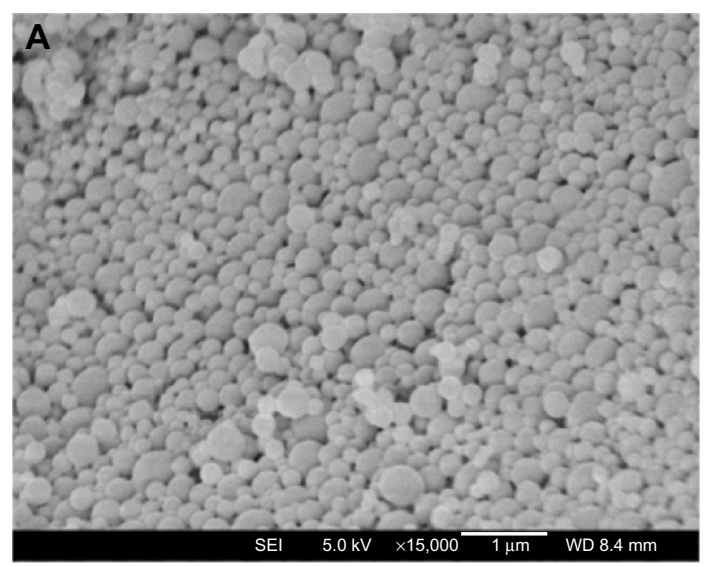

B
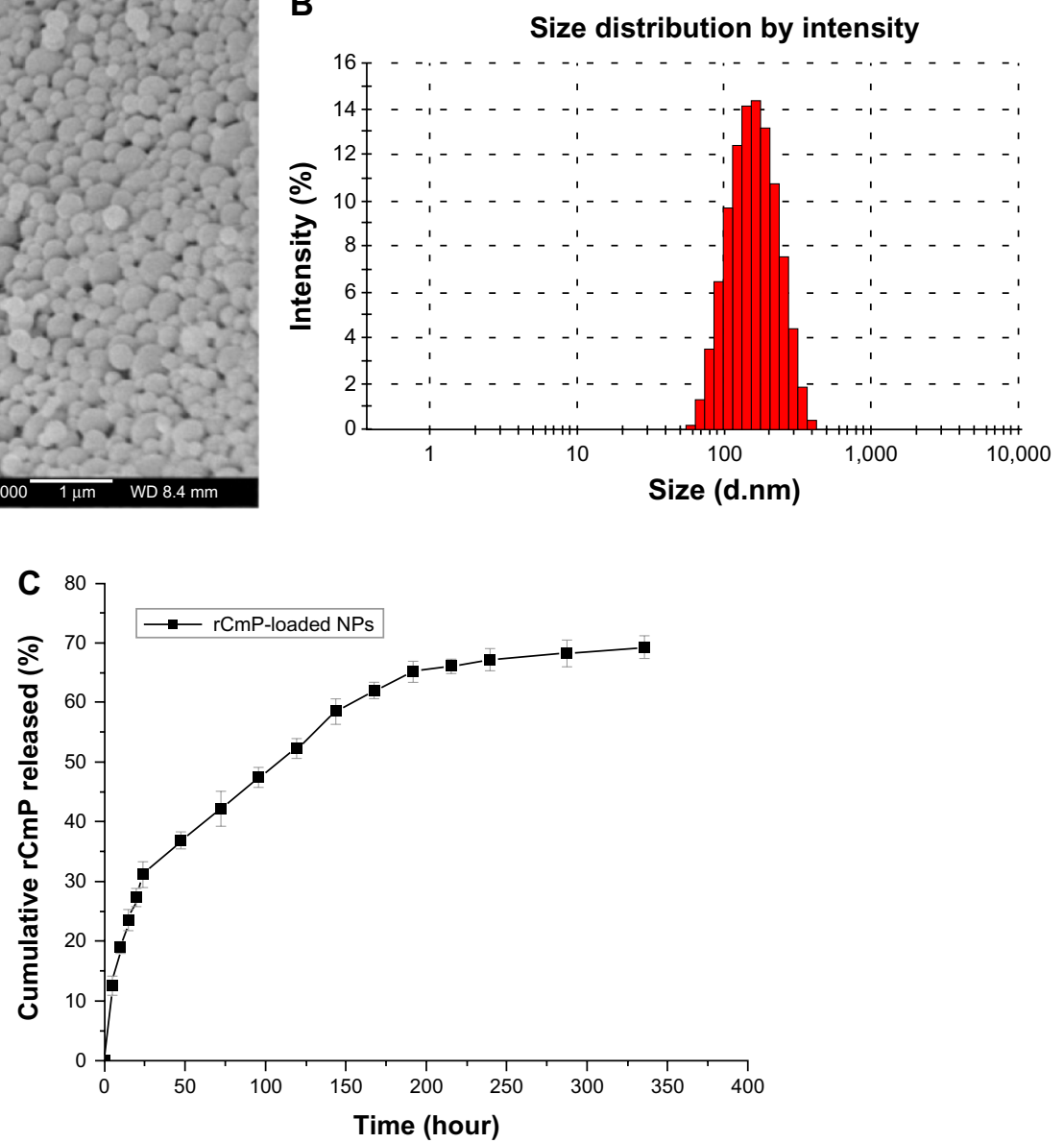

Figure 2 (A) Field emission scanning electron microscopic image, (B) dynamic light scattering spectra, and (C) in vitro cumulative protein release of rCmP-loaded PLGA nanoparticles.

Abbreviations: PLGA, poly(lactic-co-glycolic acid); rCmP, recombinant Caryota mitis profilin; NPs, nanoparticles. 
have a smooth surface within the resolution level. Further, the FESEM images demonstrated the particle size detected using a Mastersizer based on dynamic light scattering (Figure 2B).

The in vitro release research was carried out in PBS ( $\mathrm{pH}$ 7.4). The release profile of rCmP-loaded PLGA nanoparticles showed a typical biphasic release pattern (Figure 2C). The first phase was a rapid burst release in the initial 24 hours (approximately $28.3 \%$ ). This could be attributed to the amount of $\mathrm{rCmP}$ adherent to or just beneath the surface of the nanoparticles. This type of release is advantageous in terms of vaccine delivery, because it offers a primary immunization effect. The second phase consisted of constant continuous release of $\mathrm{rCmP}$ driven by diffusion through the rigid PLGA core. Release of $\mathrm{rCmP}$ reached a plateau in the following 192 hours (8 days), with a cumulative release of $65.2 \%$. Further, we found that the cumulative protein release was $69.3 \%$ within 336 hours (14 days) and no further obvious release was observed. This may be due to degradation of the encapsulated protein because of the harsh conditions formed by PLGA. A similar result was reported by Jain et al. ${ }^{19}$

\section{SDS-PAGE and Western blotting of $\mathrm{rCmP}$-loaded PLGA nanoparticles}

We used SDS-PAGE to detect changes in $\mathrm{rCmP}$ after coating with nanoparticles. The bands of PLGA-rCmP and $\mathrm{rCmP}$ are almost identical, indicating that $\mathrm{rCmP}$ and PLGA were bound without alterations (Figure 3 ). In addition, the Western blotting results (Figure 4) show that PLGA-rCmP binds to specific IgE in serum, suggesting that the activity of profilin in $\mathrm{rCmP}$-loaded PLGA nanoparticles maintained intact after being bound physically to PLGA.

\section{AHR assessment}

AHR changes were assessed by methacholine challenge 24 hours after the last allergen challenge. The results (Figure 5) show that AHR in the model group was significantly greater $(P<0.01)$ than in the normal group, and that in animals challenged by $25 \mathrm{mg} / \mathrm{mL}, 50 \mathrm{mg} / \mathrm{mL}$, and $100 \mathrm{mg} / \mathrm{mL}$ methacholine was particularly high. AHR in the PLGA-rCmP group was significantly lower than in the model group $(P<0.01)$, whereas the Penh value did not differ significantly from that of the normal group. The similar $\mathrm{AHR}$ of the $\mathrm{Al}(\mathrm{OH})_{3}$-containing $\mathrm{rCmP}$ group and the model group indicates that the rCmP-loaded PLGA nanoparticles performed better than the individual $\mathrm{rCmP}$. The blank PLGA nanoparticle control group performed similarly to the model group.

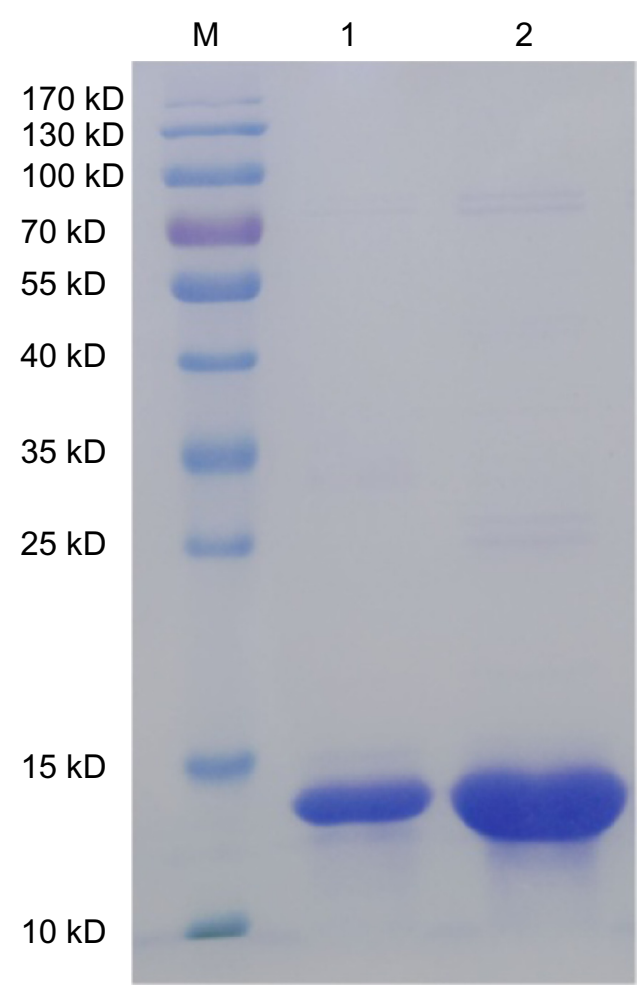

Figure 3 Sodium dodecyl sulfate polyacrylamide gel electrophoresis analysis of rCmP-loaded PLGA nanoparticles.

Notes: M, marker; I, PLGA-rCmP; 2, rCmP.

Abbreviations: PLGA, poly(lactic-co-glycolic acid); rCmP, recombinant Caryota mitis profilin.

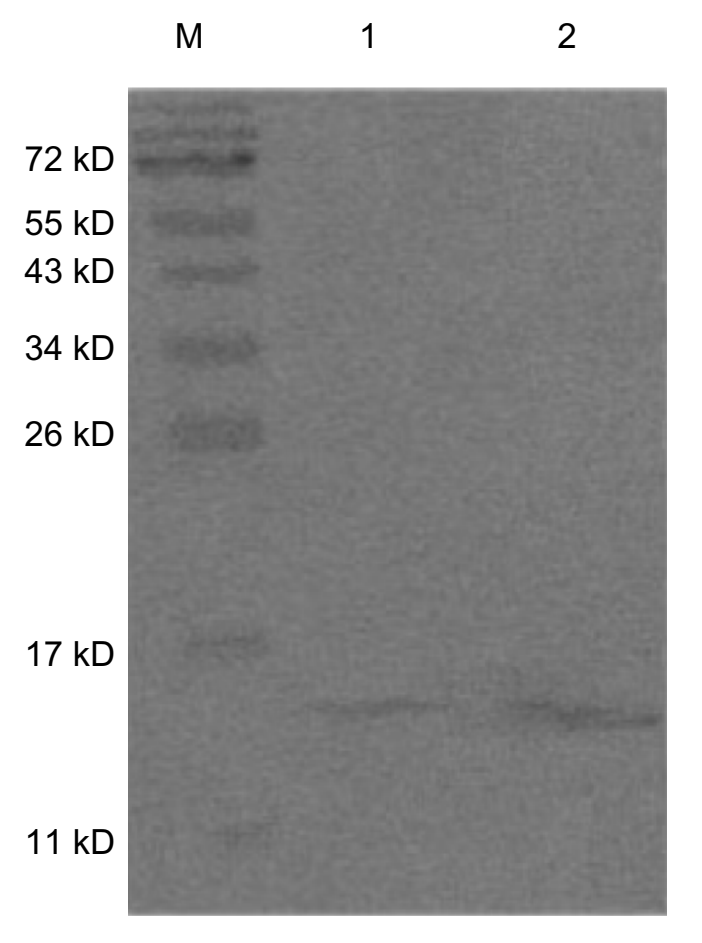

Figure 4 Western blot of rCmP-loaded PLGA nanoparticles with serum from patient.

Notes: M, marker; I, PLGA-rCmP; 2, rCmP.

Abbreviations: PLGA, poly(lactic-co-glycolic acid); rCmP, recombinant Caryota mitis profilin. 


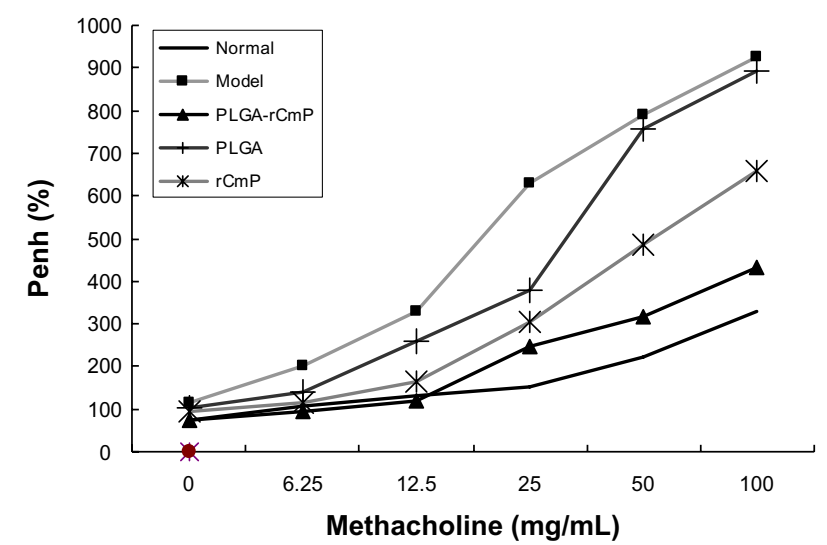

Figure 5 Airways hyperresponsiveness assay for mice.

Notes: Normal, healthy control group; model, untreated asthma model group; PLGA-rCmP, rCmP-loaded PLGA nanoparticle group; PLGA, blank PLGA nanoparticle control group; and $\mathrm{rCmP}, \mathrm{rCmP} / \mathrm{Al}(\mathrm{OH})_{3}$ vaccine group.

Abbreviations: PLGA, poly(lactic-co-glycolic acid); rCmP, recombinant Caryota mitis profilin.

\section{Determination of serum $\lg E$, $\lg G$, and $\lg G 2 a$}

Serum IgE, IgG, and IgG2a levels were measured by ELISA to evaluate the efficacy of the vaccine. The results (Figure 6) show that serum IgE levels in the model group were significantly higher than those in the normal group $(P<0.001)$. Compared with the model group, PLGA-rCmP inhibited the immune response of serum $\operatorname{IgE}$ to a greater extent than did $\mathrm{rCmP}$, given the significantly lower serum IgE levels of the two groups $(P<0.05)$. Serum IgE levels in the PLGA and model groups did not differ significantly. PLGA-rCmP and $\mathrm{rCmP}$ both elevated serum IgG and IgG2a levels significantly compared with the model group, and PLGA-rCmP induced serum IgG2a more obviously than did rCmP. Serum IgG

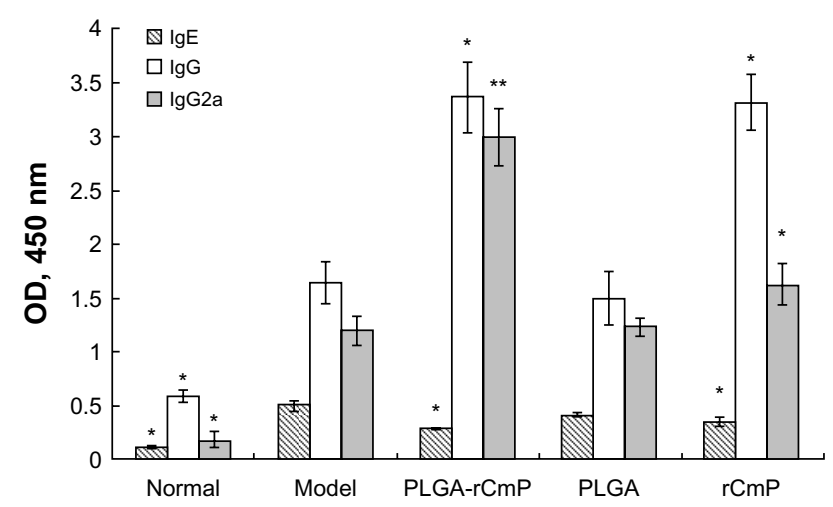

Figure 6 Allergen-specific antibodies in serum.

Notes: A450, absorbance at $450 \mathrm{~nm}$; normal, healthy control group; model, untreated asthma model group; PLGA-rCmP, rCmP-loaded PLGA nanoparticle group; PLGA, blank PLGA nanoparticle control group; $\mathrm{rCmP}, \mathrm{rCmP} / \mathrm{Al}(\mathrm{OH})_{3}$ vaccine group. $* P<0.05, * * P<0.01$ versus untreated asthma model control group. Abbreviations: PLGA, poly(lactic-co-glycolic acid); rCmP, recombinant Caryota mitis profilin; OD, optical density. and IgG2a levels in the PLGA and model groups did not differ significantly. In general, PLGA-rCmP facilitated the Th1 immune response while suppressing the Th2 response.

\section{Bronchoalveolar lavage and inflammatory cell differential counting}

Figure 7 shows that the total counts of cells and inflammatory cells in the bronchoalveolar lavage fluid sampled from the model group were significantly higher than those in the normal group $(P<0.01)$. The inflammatory cells mainly comprised neutrophils and eosinophils. The total counts for cells and inflammatory cells were significantly lower after being treated with rCmP-loaded PLGA nanoparticles and $\mathrm{rCmP}$ compared with the model group. In particular, considerably fewer cells, eosinophils, and lymphocytes were observed in the PLGA-rCmP group. In contrast, the total counts of cells and inflammatory cells in the PLGA control group do not differ from those in the model group.

\section{Cytokine assay}

IL-4, IL-10, and IFN- $\gamma$ concentrations in the splenocyte culture medium supernatant and bronchoalveolar lavage fluid detected by ELISA (Figure 8A and B) show that the model group had allergen-specific Th2 immune responses, ie, IL-4 and IFN- $\gamma$ levels, that were evidently upregulated and downregulated, respectively, compared with the normal group. After being treated with rCmP-loaded PLGA nanoparticles and rCmP, IL-4 and IFN- $\gamma$ levels were dramatically downregulated and upregulated, respectively, suggesting that treatment with the nanoparticles promoted the Th1 immune response and inhibited the $\mathrm{Th} 2 \mathrm{immune}$ response by excreting

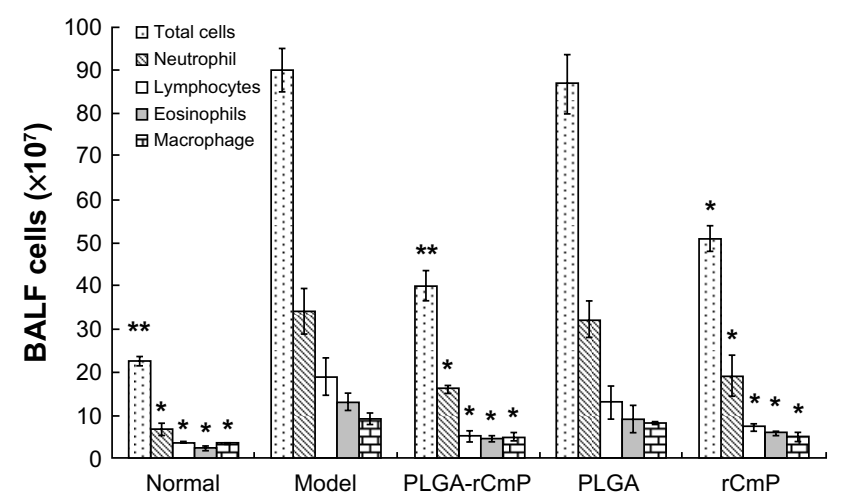

Figure 7 Differential counts of inflammatory cells in BALF.

Notes: Normal, healthy control group; model, untreated asthma model group; PLGA-rCmP, rCmP-loaded PLGA nanoparticle group; PLG, blank PLGA nanoparticle control group; $\mathrm{rCmP}, \mathrm{rCmP} / \mathrm{Al}(\mathrm{OH})_{3}$ vaccine group. $* P<0.05,{ }^{*} * P<0.01$ versus untreated asthma model control group.

Abbreviations: BALF, bronchoalveolar lavage fluid; PLGA, poly(lactic-co-glycolic acid); rCmP, recombinant Caryota mitis profilin. 

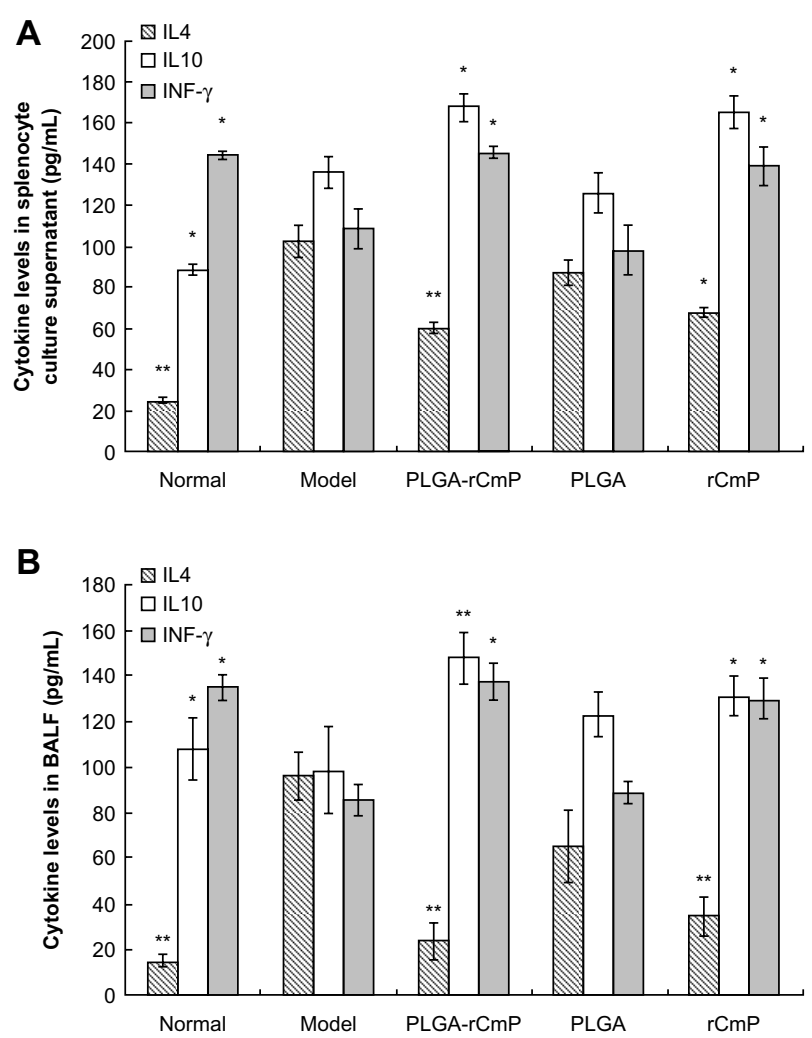

Figure 8 Cytokine production in spleen cell cultures (A) and BALF (B). Notes: Normal, healthy control group; model, untreated asthma model group; PLGA-rCmP, rCmP-loaded PLGA nanoparticle group; PLGA, blank PLGA nanoparticle control group; $r \mathrm{CmP}, \mathrm{rCmP/Al}(\mathrm{OH})_{3}$ vaccine group. $* P<0.05, * * P<0.0$ I versus untreated asthma model control group.

Abbreviations: BALF, bronchoalveolar lavage fluid; PLGA, poly(lactic-co-glycolic acid); rCmP, recombinant Caryota mitis profilin; IL-4, interleukin-4; IL-I0, interleukin- I0; IFN- $\gamma$, interferon-gamma.

more IFN- $\gamma$. In addition, IL-10 levels in the PLGA-rCmP and $\mathrm{rCmP}$ groups were higher than in the model group, which boosted anti-inflammatory and immunosuppressive functioning more effectively. The Th1/Th2 imbalance in the PLGA group was not restored, based on the concentrations of IL-4, IL-10, and IFN- $\gamma$ in the splenocyte culture medium supernatant and bronchoalveolar lavage fluid that were almost identical to those in the model group.

\section{Lung histologic analysis}

As shown in Figure 9, lung tissue structures in the normal group were well defined without discernible damage or edema, and the trachea and blood vessels were not infiltrated peripherally by inflammatory cells. However, lung tissue and bronchial structures in the model group were disturbed by internal hemorrhage and edema. The bronchial and vascular walls were thickened and infiltrated by a considerable number of inflammatory cells. Lung tissue in the PLGA-rCmP group showed histologic changes with occasional inflammatory cell infiltration similar to that seen in the normal group. Although lung tissue in the

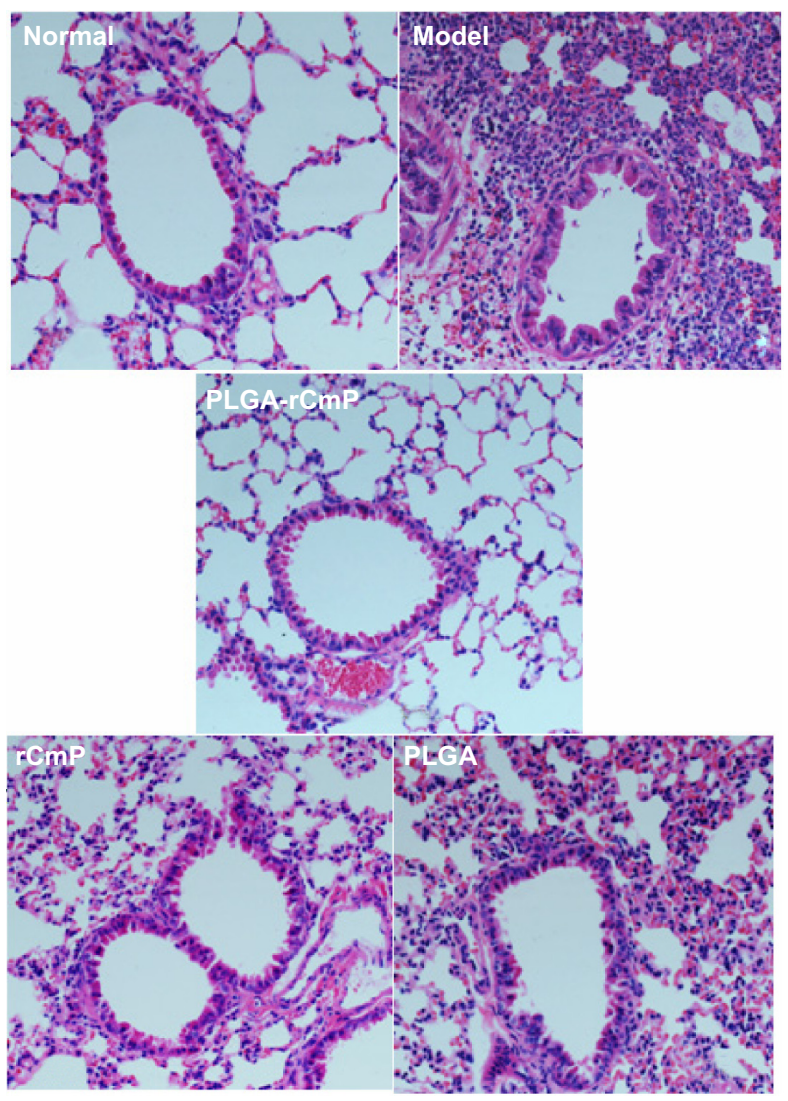

Figure 9 Formalin-fixed lung sections were stained with hematoxylin and eosin. Notes: Normal, healthy control group; model, untreated asthma model group; PLGA-rCmP, rCmP-loaded PLGA nanoparticle group; PLGA, blank PLGA nanoparticle control group; $\mathrm{rCmP}, \mathrm{rCmP/AI}(\mathrm{OH})_{3}$ vaccine group.

Abbreviations: PLGA, poly(lactic-co-glycolic acid); rCmP, recombinant Caryota mitis profilin.

rCmP group was free from edema and damage, there was markedly irregular architecture and inflammatory cell infiltration. Regardless, the histologic results of the $\mathrm{rCmP}$ group generally improved relative to those of the model group.

\section{Discussion}

Allergic airways disease mainly results from airborne pollens, that endanger over 100,000 people worldwide and $15 \%-30 \%$ of the population in developed countries. ${ }^{28,29} \mathrm{In}$ addition to nonstandardized allergen diagnostic agents and vaccines, hypoallergenic tropical pollens have seldom been investigated, unlike the enormous studies done on hyperallergenic birch, olive, and ragweed pollens. The Palmae plant, which is widely distributed in Guangdong, Hainan, Guangxi, Fujian, and some subtropical regions in the People's Republic of China, is the main source of pollen causing allergic disease in those regions. It was reported that positive skin test rates for C. mitis and Phoenix roebelenii pollens ranked highest for Palmae among 20 tested tropical plants. ${ }^{30}$ Profilin is an omnipresent panallergen in higher plants, and its highly conserved 
structure accounts for the cross-reactive nature of $\operatorname{IgE}$ antibodies against plant pollen (and plant food) profilins and their designation as panallergens. Therefore, we prepared a novel $\mathrm{rCmP}$ vaccine and explored antigen specific immunotherapy to allergic asthma. Traditional vaccines that are metabolized rapidly are subject to the environment of the organism, and cannot be delivered intact to immune system targets and maintain long-term effects. ${ }^{31}$ On the other hand, PLGA is a biodegradable polymer approved by the US Food and Drug Administration $^{32}$ and has been widely used, including in vaccines and medical industrial materials. ${ }^{33}$ Allergens encapsulated by PLGA are not exposed to environmental influences prior to being slowly released, this prolongs the therapeutic effects and stable delivery to the specific immune targets stimulating the immune responses of the host cells. ${ }^{34-36}$

In this study, mice with allergic asthma were treated with PLGA-encapsulated $\mathrm{rCmP}$ utilizing a $\mathrm{rCmP} / \mathrm{Al}(\mathrm{OH})_{3}$ vaccine group and a blank PLGA nanoparticle group as controls. We confirmed that rCmP-loaded PLGA nanoparticles and $\mathrm{rCmP}$ both mitigated allergic inflammation, and the former treatment performed more effectively in inhibiting airway inflammation, decreasing vascular permeability, and dramatically reducing the numbers of inflammatory cells, especially eosinophils in bronchoalveolar lavage fluid. Meanwhile, rCmP-loaded PLGA nanoparticles effectively inhibited generation of serum IgE and induced serum IgG2a, while facilitating binding between serum IgG and allergens and inhibiting binding between serum $\operatorname{IgE}$ and allergens. Moreover, secretion of the Th2 cytokine IL-4 and the Th1 cytokine IFN- $\gamma$ were apparently suppressed and induced by rCmP-loaded PLGA nanoparticles, respectively, indicating that $\mathrm{rCmP}$-loaded PLGA nanoparticles are a potent specific immunotherapy vaccine because of their ability to inhibit the Th2 response and stimulate the Th1 response. The augmented secretion of IL-10 after treatment with PLGA-rCmP indicates subsequent immune tolerance of allergen-specific $\mathrm{T}$ cells. Further, lung tissue in the PLGA-rCmP group showed histologic changes similar to that in the normal group in the presence of well defined structures without observable damage or edema, and the trachea and blood vessels were not infiltrated peripherally by inflammatory cells. Lung tissue in the $\mathrm{rCmP}$ group showed structural disturbance and mild infiltration by inflammatory cells, whereas the blank PLGA nanoparticles had no therapeutic benefit.

\section{Conclusion}

The rCmP-loaded PLGA nanoparticles synthesized in this study were able to prevent and treat asthma effectively by regulating Th1/Th2 equilibrium via downregulation of Th2 cytokine expression, inhibition of eosinophil differentiation, and induction of Th1 cytokine expression. PLGA acts more appropriately as a specific immunotherapy adjuvant for allergen vaccines than the conventional $\mathrm{Al}(\mathrm{OH})_{3}$ adjuvant because of its superior efficacy and longer potency, and has remarkably fewer side effects. Hence, our attempt to prepare a novel and secure PLGA-allergen nanoparticle herein will innovate the immunotherapy of allergic asthma and provide theoretical evidence for the prevention and treatment of allergic disease.

\section{Acknowledgments}

$\mathrm{ZL}$ is grateful for funding received from the Guangdong High Technology and Industrialization Project (2009B011300010), Shenzhen-Hong Kong Technology Cooperation Funding (2007), Shenzhen City Key Laboratory (SW20110010), and Shenzhen University Basic Science Research Project (201101). LM gratefully acknowledges the financial support of the National Natural Science Foundation of China (31270019, 51203085, and 81302553), Natural Science Foundation of Guangdong Province (S2012040006820, S2012010010046), Science, Technology and Innovation Commission of Shenzhen Municipality (JCYJ20120616213729920, JCYJ20120614191936420, KQC201105310021A, JC201005270308A), and the Program for New Century Excellent Talents in University (NCET11-0275).

\section{Disclosure}

The authors report no conflicts of interest in this work.

\section{References}

1. Theodoropoulos DS, Lockey RF. Allergen immunotherapy: guidelines, update, and recommendations of the World Health Organization. Allergy Asthma Proc. 2000;21(3):159-166.

2. Traidl-Hoffmann C, Kasche A, Menzel A, et al. Impact of pollen on human health: more than allergen carriers. Allergy Immunol. 2003;131(1): $1-13$.

3. Bousquet J, Van Cauwenberge P, Khaltaev N. Allergic rhinitis and its impact on asthma. J Allergy Clin Immunol. 2001;108(5):S148-S217.

4. Passalacqua G, Canonica GW. Sublingual immunotherapy: update 2006. Curr Opin Allergy Clin Immunol. 2006;6(6):449-454.

5. Lue KH, Lin YH, Sun HL, Lu KH, Hsieh JC, Chou MC. Clinical and immunologic effects of sublingual immunotherapy in asthmatic children sensitized to mites: a double-blind, randomized, placebo-controlled study. Pediatr Allergy Immunol. 2006;17(6):408-415.

6. Ebner C, Siemann U, Bohle B, et al. Immunological changes during specific immunotherapy of grass pollen allergy: reduced lymphoproliferative responses to allergen and shift from Th2 to Th1 in T-cell clones specific for Ph1 p 1, a major grass pollen allergen. Clin Exp Allergy. 1997;27(9): 1007-1015.

7. Secrist H, Chelen CJ, Wen Y, Marshall JD, Umetsu DT. Allergen immunotherapy decreases interleukin 4 production in CD4 T cells from allergic individuals. J Exp Med. 1993;178(6):2123-2130. 
8. Beusquet J, Lockey R, Malling HJ. WHO Position Paper. Allergen immunotherapy: therapeutic vaccines for allergic diseases. $J$ Allergy Clin Immunol. 1998;102(4 Pt 1):558-562.

9. Peek LJ, Middaugh CR, Berkland C. Nanotechnology in vaccine delivery. Adv Drug Deliv Rev. 2008;60(8):915-928.

10. Zhang Z, Guo Y, Feng SS. Nanoimmunotherapy: application of nanotechnology for sustained and targeted delivery of antigens to dendritic cells. Nanomedicine. 2012;7(1):1-4.

11. Hong Z, Zhang P, Liu A, Chen L, Chen X, Jing X. Composites of poly(lactide-co-glycolide) and the surface modified carbonated hydroxyapatite nanoparticles. J Biomed Mater Res. 2007;81(3):515-522.

12. Dinarvand R, Sepehri N, Manoochehri S, Rouhani H, Atyabi F. Polylactide-co-glycolide nanoparticles for controlled delivery of anticancer agents. Int J Nanomedicine. 2011;6:877-895.

13. Klose D, Siepmann F, Elkharraz K, Siepmann J. PLGA-based drug delivery systems: importance of the type of drug and device geometry. Int J Pharm. 2008;354(1-2):95-103.

14. Dorozhkin SV. Calcium orthophosphate based biocomposites and hybrid materials. J Mater Sci. 2009;44(9):2343-2387.

15. Ren J, Zhao P, Ren T, Gu S, Pan K. Poly (D,L-lactide)/nanohydroxyapatite composite scaffolds for bone tissue engineering and biocompatibility evaluation. J Mater Sci Mater Med. 2008;19(3): 1075-1082.

16. Scholl I, Weissenbock A, Forster-Waldl E, et al. Allergen-loaded biodegradable poly(D,L-lactic-co-glycolic) acid nanoparticles downregulate an ongoing Th2 response in the BALB/c mouse model. Clin Exp Allergy. 2004;34(2):315-321.

17. Santos A, Van Ree R. Profilins: mimickers of allergy or relevant allergens? Int Arch Allergy Immunol. 2011;155(3):191-204.

18. Liu XY, Wu YL, Liu ZG, Meng G, Zhang HY. Cloning, expression and immunocharacterization of panallergen profilin from Caryota mitis pollen. J Trop Med. 2007;7(7):618-622.

19. Jain AK, Goyal AK, Gupta PN, et al. Synthesis, characterization and evaluation of novel triblock copolymer based nanoparticles for vaccine delivery against hepatitis B. J Control Release. 2009;136(2): 161-169.

20. Buske J, König C, Bassarab S, Lamprecht A, Mühlau S, Wagner KG. Influence of PEG in PEG-PLGA microspheres on particle properties and protein release. Eur J Pharm Biopharm. 2012;81(1):57-63.

21. Yu HQ, Li XH, Liu ZG, et al. Sublingual immunotherapy efficacy of Dermatophagoides farinae vaccine in a murine asthma model. Int Arch Allergy Immunol. 2009;152(1):41-48.

22. Winkler B, Baier K, Wagner S, et al. Mucosal tolerance as therapy of type I allergy: intranasal application of recombinant Bet $\mathrm{v}$ 1, the major birch pollen allergen, leads to the suppression of allergic immune responses and airway inflammation in sensitized mice. Clin Exp Allergy. 2002;32(1):30-36.
23. Lu M, Dawicki W, Zhang X, Huang H, Nayyar A, Gordon JR. Therapeutic induction of tolerance by IL-10-differentiated dendritic cells in a mouse model of house dust mite-asthma. Allergy. 2011;66(5): 612-620.

24. Marazuela EG, Rodríguez R, Barber D, Villalba M, Batanero E. Hypoallergenic mutants of Ole e 1, the major olive pollen allergen, as candidates for allergy vaccines candidates for allergy vaccines. Clin Exp Allergy. 2007;37(2):251-260.

25. Florence AT. Nanoparticle uptake by the oral route: fulfilling its potential? Drug Discov Today Technol. 2005;2(1):75-81.

26. Yan F, Zhang C, Zheng Y, et al. The effect of Poloxamer 188 on nanoparticle morphology, size, cancer cell uptake, and cytotoxicity. Nanomedicine. 2010;6(1):170-178.

27. Huang LQ, Chen H, Zheng Y, et al. Nanoformulation of D-a-tocopheryl polyethylene glycol 1000 succinate-b-poly ( $\varepsilon$-caprolactone- ranglycolide) diblock copolymer for breast cancer therapy. Integr Biol (Camb). 2011;3(10):993-1002.

28. Roitt I, Brostoff J, Male D. Immunology. 5th ed. Philadelphia, PA: Mosby; 1998.

29. Vieths S, Scheurer S, Ballmer-Weber B. Current understanding of crossreactivity of food allergens and pollen. Ann N Y Acad Sci. 2002;964: 47-68.

30. Meng G, Li C, Cai Q, Xie C. Survey on pollen sensitization of tropic plants in Haikou. J Clin Otorhinolaryngol (China). 2005;19(22): 1044-1045. Chinese.

31. Martínez-Gómez JM, Johansen P, Erdmann I, Senti G, Crameri R, Kündig TM. Intralymphatic injections as a new administration route for allergen-specific immunotherapy. Int Arch Allergy Immunol. 2009;150(1):59-65.

32. Mohaghegh M, Tafaghodi M. Dextran microspheres could enhance immune responses against PLGA nanospheres encapsulated with tetanus toxoid and Quillajasaponins after nasal immunization in rabbit. Pharm Dev Technol. 2011;16(1):36-43.

33. Schnieders J, Gbureck U, Thull R, Kissel T. Controlled release of gentamicin from calcium phosphate-poly(lactic acid-co-glycolic acid) composite bone cement. Biomaterials. 2006;27(23):4239-4249.

34. Zhao K, Li GX, Jin YY, et al. Preparation and immunological effectiveness of a Swine influenza DNA vaccine encapsulated in PLGA microspheres. J Microencapsul. 2010;27(2):178-186.

35. Patel VF, Liu F, Brown MB. Advances in oral transmucosal drug delivery. J Control Release. 2011;153(2):106-116.

36. Kalkanidis M, Pietersz GA, Xiang SD, et al. Methods for nano-particle based vaccine formulation and evaluation of their immunogenicity. Methods. 2006;40(1):20-29.
International Journal of Nanomedicine

\section{Publish your work in this journal}

The International Journal of Nanomedicine is an international, peerreviewed journal focusing on the application of nanotechnology in diagnostics, therapeutics, and drug delivery systems throughout the biomedical field. This journal is indexed on PubMed Central, MedLine, CAS, SciSearch ${ }^{\circledR}$, Current Contents ${ }^{\circledR} /$ Clinical Medicine,

\section{Dovepress}

Journal Citation Reports/Science Edition, EMBase, Scopus and the Elsevier Bibliographic databases. The manuscript management system is completely online and includes a very quick and fair peer-review system, which is all easy to use. Visit http://www.dovepress.com/ testimonials.php to read real quotes from published authors. 